
\title{
3 Research Square \\ The feasibility of transfer learning for differentiation H1N1 Influenza from COVID-19 on chest CT
}

Houman Sotoudeh ( $\nabla$ hsotoudeh@uabmc.edu )

University of Alabama at Birmingham

\section{Baharak Tasorian}

Arak University of Medical Sciences

Seyed Mohsen Tabatabaei

Arak University of Medical Sciences

\section{Ehsan Sotoudeh}

Red Crescent Hospital in Dubai

\section{Abdollatif Moini}

Arak University of Medical Sciences

\section{Research Article}

Keywords: COVID-19, Influenza, Artificial intelligence, Machine learning

Posted Date: July 14th, 2020

DOI: https://doi.org/10.21203/rs.3.rs-40907/v1

License: (c) (1) This work is licensed under a Creative Commons Attribution 4.0 International License. Read Full License 


\section{Abstract}

Objectives: It is unlikely that by fall and winter of 2020, standard vaccine or treatment is available for COVID-19 infection. In this period, differentiation between COVID-19 and Influenza induced pneumonia will be critical for patient management. To develop an automated platform to perform this task, artificial intelligence models were developed by using the transfer learning techniques on chest CT.

Methods: Chest CT images from known cases of COVID-19, H1N1 Influenza induced pneumonia (before December 2019), and normal chest CTs were collected. Different pre-trained Convolutional Neural Networks (CNN) models, including VGG 16, VGG 19, ResNet-50, Wide ResNet, InceptionV3, and SqueezNet were fine-tuned on this data set. $60 \%$ of the dataset was used for training, $20 \%$ for validation, and $20 \%$ for test the final models. Accuracy, Precision, Recall and F1 score of each model were calculated.

Results: For differentiation of COVID-19 pneumonia versus H1N1 Influenza pneumonia versus normal CTs, the ResNet-50 (accuracy above 92\%) outperformed other models followed by InceptionV3 and wide ResNet.

Conclusions: The pre-trained image classification Al models are feasible to be fine-tuned and used for differentiation COVID-19 versus H1N1 Influenza pneumonia. In this context, ResNet-50 and then InceptionV3 architectures appear more promising and are suitable start points for further development. We share the source code and trained models in the supplement of this manuscript to be used by other researchers for further development.

\section{Key Points}

Differentiation between COVID-19 and Influenza is a challenging task based on clinical and laboratory findings.

The development of automated platforms based on Convolutional Neural Networks and transfer learning techniques are feasible to do this task.

ResNet-50 and InceptionV3 are promising pre-trained models and can be used as start points.

\section{Introduction}

\section{COVID-19}

A new form of respiratory infection was detected in Wuhan city, China, in December 2019. In less than one month, the pathogen was recognized as a novel type of Coronavirus. It was named as "severe acute respiratory syndrome coronavirus 2" (SARS-CoV-2), and the resultant infection was called COVID-19. Despite robust international measurements and quarantines in many countries, this form of infection became a pandemic in less than three months. By July 24,2020, about 9.3 million infections and 478000 death have been reported in 188 countries because of COVID-19 (1-4). The SARS-CoV-2 is an enveloped 
single-stranded RNA virus that attaches to the angiotensin-converting enzyme 2 of airways with a duration of infectiousness of 3 days before the onset of symptoms until clearance of the virus. The incubation time is $2-14$ days. No vaccine or standard treatment is available for COVID-19 at this time. It is unknown if the COVID-19 prevalence is different in different seasons (5).

\section{Influenza}

Influenza infection is one of the common forms of respiratory viral infection. It has been estimated that each year between 291,000 to 645,000 death happens because of the influenza infection in the world (6, 7). There are four forms of influenza virus, including $A, B, C$, and D. Usually, the seasonal Influenza is caused by type A and B (8). H1N1 Influenza is one of the worst subtypes of influenza infection and caused two pandemics in 1918 and 2009 with 50 million and over 280000 death, respectively $(9,10)$. The influenza virus is an enveloped single-stranded RNA virus that attaches to the $\mathrm{N}$-acetyl neuraminic acid in the airways. Its distribution is via droplets. Duration of infectiousness is from 1 day before illness to as long as severe symptoms persist with an incubation time of 1-4 days. There are several approved vaccines and treatments for the Influenza virus, and its prevalence is seasonal (5).

Clinical differentiation between COVID-19 and Influenza is difficult. There is a substantial overlap between the clinical manifestations of Influenza and COVID-19. Fever, cough, expectoration, and dyspnea are the main manifestation of these two infections. Other clinical presentations of these viral infections are headache, sore throat, chest pain, fatigue, myalgia, nausea, vomiting, and diarrhea. Cough and expectoration have been reported more commonly in Influenza. The prevalence of other clinical findings is similar in these two diseases (6).

Also, there is a substantial overlap in laboratory findings in these two diseases. Lymphopenia elevated Creactive protein, and erythrocyte sedimentation rate levels have been reported in both diseases without significant differences (6). The Procalcitonin level is also not significantly different between these two infections (6).

\section{Reverse transcription-polymerase chain reaction ( $R T-P C R)$}

While the rapid RT-PCR assay is a very robust technique for diagnosis of Influenza A and B with a sensitivity of $98 \%$ and specificity of $99 \%$ (11), the same thing is not true for COVID-19 PCR. The accuracy of PCR in COVID-19 depends on the time and technique of sampling. Its false negative result is very high in the incubation phase ( $100 \%$ false-negative five days before initial symptoms, $67 \%$ false-negative one day before initial symptoms). PCR is falsely negative in $38 \%$ of patients on the day of initial symptoms. Its false negative rate is $20 \%$ three days after initial symptoms and $21 \%$ four days after initial symptoms (12).

\section{CT scan}

Currently, there are a few studies regarding the role of medical imaging for the differentiation of these two infections. The disease burden of COVID-19 on chest CT is reported to be higher than the Influenza A (CT 
score of 13 versus 6) (6). Also, it has been reported that the frequencies of bronchiectasis, pleural effusions, linear opacities, crazy-paving opacities, and vascular enlargement within the pulmonary lesions are different in COVID-19 and Influenza A pneumonia and can be potentially be used for differentiation (6). In another study, the COVID-19 patients had more rounded opacities and interlobular septal thickening on chest CT but less pulmonary nodules, tree-in-bud opacities and pleural effusion in comparison to patients with Influenza A and B (13). Even though the most CT manifestations of viral infection are nonspecific, the preliminary reports are promising about the role of $\mathrm{CT}$ to differentiate these two infections.

\section{Fall and winter of 2020}

It is unlikely that the vaccine and standard treatment for the COVID-19 are available by fall and winter of 2020. In this period, seasonal flu can be superimposed on the COVID-19 pandemic. Differentiation between these two infections is critical for patient management. Given the facts mentioned above and substantial overlap between clinical and laboratory presentations of COVID-19 and Influenza, CT would be critical for this task. Differentiation of COVID-19 versus Influenza on chest CT is challenging for medical centers without experienced chest radiologists. In this study, we tested the feasibility of automated diagnostic techniques based on transfer learning Al on chest CT images. Having such platforms can help physicians without chest imaging experience during the fall and winter of 2020.

\section{Materials And Methods}

This study was approved by the ethical committee of Arak University of Medical Sciences (IR.ARAKMU.REC.1398.339). Medical data and Images from patients with diagnoses of COVID-19 and H1N1 Influenza were reviewed by a pulmonologist with 25 years of experience and a radiologist with 12 years of experience. Cases with motion artifacts, poor image quality, and chronic lung disease were excluded. Medical data and chest CT images from 72 patients with clinical and PCR diagnosis of COVID19 were collected from February 2020 to May 2020 from the tertiary referral centers of the mentioned university. Also, medical data and chest CT images from 39 patients with PCR positive H1N1 Influenza induced pneumonia were collected from 2017 to December 2019 at the same medical centers. The Influenza cases were collected before December 2019 to make sure that there is no concurrent infection of COVID-19 and Influenza. Finally, 26 normal chest CT studies were also collected. All CT scans were performed by the standard chest protocol (MA: 24-40, KVp: 100-110, Slice thickness: less than $1.5 \mathrm{~mm}$, Pitch factor: 0.8 and Matrix: 512X512). Axial slices were used in this study. Using the ImageJ platform, the grayscale images were converted to RBG format. All slices of COVID-19 and Influenza cohorts were reviewed by the same radiologist, and slices without visible pathology were deleted. Then in the normal cohort, one slice out of nine slices and in COVID-19 and Influenza cohorts, one slice out of any four slices were selected and used for training (this technique was performed to feed the models with different slices and help them to capture useful information). Each axial slice was divided vertically into right and left hemithorax. Augmentation was also performed by 30 degrees of rotation, 0.2 rescalings ( 0.2 width shift range, 0.2 height shift range, and 0.2 share range). The final labeled images were uploaded to different 
pre-trained CNN models. Overall, 12744 images were used, including 2503 COVID-19, 3035 Influenza, and 7206 normal CT images. Images were resized to be acceptable to each model. The pre-trained models were VGG 16, VGG 19, ResNet-50, Wide ResNet, InceptionV3, and SqueezNet. Each model was pre-trained on 1000 image classes of ImageNet. For ResNet-50, four models were developed by $0 \%, 20 \%, 30 \%$ and $40 \%$ trainability. For InceptionV3, three models were developed by $0 \%, 20 \%$, and $30 \%$ trainability. Other models were developed by $0 \%$ and $20 \%$ trainability. $60 \%$ of data was used for training, $20 \%$ for validation, and $20 \%$ for test. The training was done on the Deep Learning Studio (14). The output of each pre-trained model was flattened using a flatten layer and then was fed to a dense layer with three classes output representing the prediction for normal, COVID-19, and Influenza. Training process was similar in all models (Number of Epoch: 10, Batch size: 32, Loss Function: categorical crossentropy, Optimizer: Adam, beta 1: 0.9 , beta 2: 0.999 , decay: 0 and Ir: 0.001$)$. The final models were tested on the test cohort (20\% of unseen data). The accuracy, precision, recall, and F1 score of each model were calculated and reported.

\section{Results}

The COVID-19 cohort consisted of 38 male and 34 female, the mean age of 60.9 years, and with the mean time interval between the initial symptoms and CT of 4.37 days. The Influenza cohort consisted of 20 males and 19 females, the mean age of 62.4 , and the mean time interval between initial symptoms and CT of 5.41 days. By our pipe-line design, the ResNet-50, InceptionV3, Wide ResNet with various trainablities, and VGG 19 with $0 \%$ trainability were able to capture useful information and performed well on the validation and test cohort. The ResNet-50 with 20 and $30 \%$ trainability had the best performance to differentiate COVID-19 from Influenza induced pneumonia. The results of each model are summarized in Table 1. 


\begin{tabular}{|c|c|c|c|c|c|}
\hline Model & Prediction & Accuracy & Precision & Recall & F1Score \\
\hline \multirow[t]{3}{*}{ ResNet50-20\% (supplement $1 \& 2$ ) } & Normal & $95.76 \%$ & 0.97 & 0.96 & 0.96 \\
\hline & COVID-19 & $96.78 \%$ & 0.97 & 0.88 & 0.93 \\
\hline & Influenza & $92.54 \%$ & 0.79 & 0.88 & 0.83 \\
\hline \multirow{3}{*}{$\begin{array}{l}\text { ResNet50-0\% } \\
\text { (supplement 3\&4) }\end{array}$} & Normal & $82.5 \%$ & 0.88 & 0.82 & 0.85 \\
\hline & COVID-19 & $91.99 \%$ & 0.93 & 0.74 & 0.82 \\
\hline & Influenza & $80.3 \%$ & 0.39 & 0.65 & 0.49 \\
\hline \multirow[t]{3}{*}{ ResNet50-30\% (supplement 5\&6) } & Normal & $94.11 \%$ & 0.98 & 0.92 & 0.95 \\
\hline & COVID-19 & $97.17 \%$ & 1.0 & 0.88 & 0.93 \\
\hline & Influenza & $91.29 \%$ & 0.69 & 0.92 & 0.79 \\
\hline \multirow{3}{*}{$\begin{array}{l}\text { ResNet50-40\% } \\
\text { (supplement 7\&8) }\end{array}$} & Normal & $95.37 \%$ & 0.94 & 0.98 & 0.96 \\
\hline & COVID-19 & $96.94 \%$ & 0.97 & 0.88 & 0.92 \\
\hline & Influenza & $92.31 \%$ & 0.84 & 0.83 & 0.83 \\
\hline \multirow{3}{*}{$\begin{array}{l}\text { InceptionV3-20\% } \\
\text { (supplement 9\&10) }\end{array}$} & Normal & $86.54 \%$ & 0.98 & 0.82 & 0.89 \\
\hline & COVID-19 & $96.7 \%$ & 0.98 & 0.87 & 0.92 \\
\hline & Influenza & $83.79 \%$ & 0.38 & 0.88 & 0.53 \\
\hline \multirow{2}{*}{$\begin{array}{l}\text { InceptionV3-30\% } \\
\text { (supplement } 11 \& 12 \text { ) }\end{array}$} & Normal & $77.71 \%$ & 1.0 & 0.71 & 0.83 \\
\hline & COVID-19 & $96.82 \%$ & 1.0 & 0.86 & 0.93 \\
\hline
\end{tabular}




\begin{tabular}{|c|c|c|c|c|c|}
\hline & Influenza & $74.76 \%$ & 0 & 0 & 0 \\
\hline \multirow[t]{3}{*}{ InceptionV3-0\% (supplement $13 \& 14$ ) } & Normal & $89.72 \%$ & 0.94 & 0.89 & 0.91 \\
\hline & COVID-19 & $95.6 \%$ & 0.97 & 0.83 & 0.89 \\
\hline & Influenza & $86.89 \%$ & 0.59 & 0.82 & 0.69 \\
\hline \multirow{3}{*}{$\begin{array}{l}\text { Wide ResNet-20\% } \\
\text { (supplement 15\&16) }\end{array}$} & Normal & $85.4 \%$ & 0.88 & 0.86 & 0.87 \\
\hline & COVID-19 & $95.53 \%$ & 0.86 & 0.92 & 0.89 \\
\hline & Influenza & $80.93 \%$ & 0.59 & 0.60 & 0.59 \\
\hline \multirow{3}{*}{$\begin{array}{l}\text { Wide ResNet-0\% } \\
\text { (supplement 17\& 18) }\end{array}$} & Normal & $83.36 \%$ & 0.94 & 0.80 & 0.86 \\
\hline & COVID-19 & $96.98 \%$ & 0.97 & 0.89 & 0.93 \\
\hline & Influenza & $80.34 \%$ & 0.34 & 0.65 & 0.45 \\
\hline \multirow[t]{3}{*}{ VGG19-0\% (supplement $19 \& 20$ ) } & Normal & $86.73 \%$ & 0.94 & 0.84 & 0.89 \\
\hline & COVID-19 & $95.37 \%$ & 0.99 & 0.81 & 0.89 \\
\hline & Influenza & $83.67 \%$ & 0.45 & 0.79 & 0.57 \\
\hline
\end{tabular}

Table1. The performance of different models in the classification of chest CT images presented by the accuracy, precision, recall, and F1 score in the test cohort.

\section{Discussion}

The COVID-19 disease, a novel infective pandemic is now one of the worst challenges the modern medicine has ever encountered. It became a pandemic in less than three months involving the entire world. It is unlikely that an effective treatment or vaccine would be available in the near future. This fact 
would be challenging in the fall and winter of 2020 when the seasonal Influenza outbreak may be superimposed on the COVID-19 pandemic. There is substantial clinical and laboratory overlap between these two diseases. To make things even more complicated, the current standard of care (PCR tests) is not perfect for the diagnosis of COVID-19. In this context, imaging (chest CT) may play a critical role. The chest CT is the backbone of medical imaging in such respiratory infections. In one study by Yin et al., the prevalence of bronchiectasis, pleural effusion, linear opacities, crazy-paving sign, vascular enlargement, and pleural thickening were statistically different in these two infections. However, the distribution of lesions, ground-glass opacities, consolidations, nodular opacities, bronchial thickening, lymphadenopathy, pericardial effusion, and air-bronchogram were not statistically different in these two infections (6). Despite these findings, differentiation between Influenza and COVID-19 remains challenging because the CT manifestations of these viral infections are nonspecific. Diagnosis would be even more challenging in medical centers without expert chest radiologists. Given the facts mentioned above, having an automated diagnostic system to differentiate COVID-19 versus Influenza on chest CT images may improve the diagnostic accuracy and patients' management. These automated platforms would be an essential part of the diagnosis process, especially in the areas without access to expert radiologists and while the pandemic overload can overwhelm the medical staff.

Recently, the state of the art Al models has been used for image classification. In this context, the convolutional neural network (CNN) models are very promising. Different CNN-based models even were able to achieve accuracy better than humans in image classification. The common problem during the application of CNN models for medical imaging is the size of datasets. The modern Al models, including the CNNs, are data-hungry algorithms. To achieve accuracy equal to humans or even more, these models need thousands to million images to be trained. Having datasets of medical images containing millions of samples is challenging in medicine. This problem was partially solved by transfer learning techniques. In transfer learning, the CNN model is not developed from scratch. Instead, a pre-trained model is used. The pre-trained models usually are trained on large datasets of million images (mostly on ImageNet dataset containing millions of non-medical images). Such a pre-trained model then retrained (fine-tuned) on a small dataset of medical images. The idea behind such transfer learning is the fact that basic tasks of image classification (such as edge detection, vertical and horizontal lines, etc...) can be learned from the non-medical datasets. These pre-trained models are then fine-tuned over medical images and can achieve acceptable performance on small medical datasets (15). A similar concept is true in our study. Here we were able to train CNN models for the image classification while our dataset is small (137 subject and 12744 images, which is considered to be a small dataset for CNN models). We detected the high performance in ResNet-50 and Inception. We caught the highest accuracy in ResNet-50 with the trainability of 20 and $30 \%$. The accuracy and precision of ResNet-50 $30 \%$ was $97.17 \%$ and $100 \%$ to diagnose COVID-19. The accuracy of the same model was $94.1 \%$ and $91.2 \%$ for the diagnosis of the normal and Influenza CTs, respectively. Such a model can be an added value to daily clinical practice. Our models have been trained to predict normal, COVID-19, or Influenza as their output.

Recently there have been a few case series of co-infection of COVID-19+Influeza $(16,17)$. It is unclear what would be the output of our models while they see a case of co-infection of COVID-19+Influeza.It is 
expected that such a co-infection may encounter more frequently by the end of 2020 .

The source code and the trained models are provided in the supplements and can be used by other researchers for further research projects (they are not approved for clinical applications). It must be noted that the provided models have been trained on the RBG images, so grayscale CT images must be converted to RBG format to be feed to the models. Also, they work only on axial images. They have been trained on the images of the lung parenchyma, so the neck base and upper abdomen images must be deleted before the implementation of these models. Lastly, for data augmentation, these models have been trained on the right or left hemithorax, so for deployment, they must be fed by divided axial slices. The input of models is an axial image of each hemithorax in the lung window, and the output would be a prediction about normal, H1N1 Influenza, or normal hemithorax.

In conclusion, the development of an automated diagnostic platform to differentiate COVID-19 from H1N1 Influenza or normal chest CTs is feasible with acceptable accuracy. Given our knowledge, our platform is the first solution for this task. Such a platform may enhance the efficiency of the radiologist with limited chest imaging experience. The ResNet-50 and then InceptionV3 were promising for this task in our study and are suitable start point to develop an automated platform.

\section{List Of Abbreviations}

SARS-CoV-2: severe acute respiratory syndrome coronavirus 2; RNA: Ribonucleic acid; RT-PCR: Reverse transcription-polymerase chain reaction; CNN: convolutional neural network.

\section{Declarations}

The authors have no conflict of interest.

Participant consent - All participant consented to participate. All patients received standard of care treatment according the treatment protocols of mentioned university. The study was supervised and approved by the ethical committee.

\section{References}

1. Zhu N, Zhang D, Wang W, Li X, Yang B, Song J, et al. A Novel Coronavirus from Patients with Pneumonia in China, 2019. N Engl J Med. 2020;382(8):727-33.

2. Kanne JP. Chest CT Findings in 2019 Novel Coronavirus (2019-nCoV) Infections from Wuhan, China: Key Points for the Radiologist. Radiology. 2020;295(1):16-7.

3. Pan Y, Guan H, Zhou S, Wang Y, Li Q, Zhu T, et al. Initial CT findings and temporal changes in patients with the novel coronavirus pneumonia (2019-nCoV): a study of 63 patients in Wuhan, China. Eur Radiol. 2020;30(6):3306-9. 
4. Chung M, Bernheim A, Mei X, Zhang N, Huang M, Zeng X, et al. CT Imaging Features of 2019 Novel Coronavirus (2019-nCoV). Radiology. 2020;295(1):202-7.

5. Wormser GP. COVID-19 versus seasonal influenza 2019-2020: USA. Wien Klin Wochenschr. 2020.

6. Yin Z, Kang Z, Yang D, Ding S, Luo H, Xiao E. A Comparison of Clinical and Chest CT Findings in Patients With Influenza A (H1N1) Virus Infection and Coronavirus Disease (COVID-19). AJR Am J Roentgenol. 2020:1-7.

7. Iuliano AD, Roguski KM, Chang HH, Muscatello DJ, Palekar R, Tempia S, et al. Estimates of global seasonal influenza-associated respiratory mortality: a modelling study. Lancet. 2018;391(10127):1285-300.

8. Voskarides K, Christaki E, Nikolopoulos GK. Influenza Virus-Host Co-evolution. A Predator-Prey Relationship? Front Immunol. 2018;9:2017.

9. Shoubaki LA. Surveillance snapshot: Summary of the Department of Defense Global Respiratory Pathogen Surveillance Program, 2017-2018 influenza season. MSMR. 2018;25(10):22.

10. Saunders-Hastings PR, Krewski D. Reviewing the History of Pandemic Influenza: Understanding Patterns of Emergence and Transmission. Pathogens. 2016;5(4).

11. Maignan M, Viglino D, Hablot M, Termoz Masson N, Lebeugle A, Collomb Muret R, et al. Diagnostic accuracy of a rapid RT-PCR assay for point-of-care detection of influenza A/B virus at emergency department admission: A prospective evaluation during the 2017/2018 influenza season. PLoS One. 2019;14(5):e0216308.

12. Kucirka LM, Lauer SA, Laeyendecker O, Boon D, Lessler J. Variation in False-Negative Rate of Reverse Transcriptase Polymerase Chain Reaction-Based SARS-CoV-2 Tests by Time Since Exposure. Ann Intern Med. 2020.

13. Liu M, Zeng W, Wen Y, Zheng Y, Lv F, Xiao K. COVID-19 pneumonia: CT findings of 122 patients and differentiation from influenza pneumonia. Eur Radiol. 2020.

14. https://deepcognition.ai/. Deep Learning Studio 3.0. Deep Cognition Inc, Irving, Texas, USA, 2020.

15. Mohajerani P, Sotoudeh H. Essentials of Al techniques. 2020.

16. Konala VM, Adapa S, Naramala S, Chenna A, Lamichhane S, Garlapati PR, et al. A Case Series of Patients Coinfected With Influenza and COVID-19. J Investig Med High Impact Case Rep. 2020;8:2324709620934674.

17. Ozaras R, Cirpin R, Duran A, Duman H, Arslan O, Bakcan Y, et al. Influenza and COVID-19 Co-infection: Report of 6 cases and review of the Literature. J Med Virol. 2020.

\section{Supplementary Files}

This is a list of supplementary files associated with this preprint. Click to download.

- 1ResNet200NLYforresearch.docx

- 2ResNet200NLYforresearch.yaml 
- 3ResNet0ONLYforresearch.docx

- 4ResNet0ONLYforresearch.yaml

- 5ResNet300NLYforresaech.docx

- 6ResNet300NLYforresearch.yaml

-7ResNet400NLYforresearch.docx

- 8ResNet400NLYforresearch.yaml

- 9InceptionV3200NLYforresearch.docx

- 10InceptionV3200NLYforresearch.yaml

- 11 InceptionV3300NLYforresearch.docx

- 12 InceptionV3300NLY forresearch.yaml

- 13InceptionV300NLYforresearch.docx

- 14InceptionV300NLYforresearch.yaml

- 15WideResNet200NLYforresearch.docx

- 16WideResNet200NLYforresearch.yaml

- 17WideResnet0ONLYforresearch.docx

- 18WideResNet0ONLYforresearch.yaml

- 19VGG1900NLYforresearch.docx

- 20VGG1900NLYforresearch.yaml 